\title{
Searching for gamma-ray binaries using GOSC and Gaia DR2
}

\section{Ayan-Míguez}

Universitat de Barcelona, ICCUB, Barcelona, Spain

E-mail: iayan@icc.ub.edu

\section{Ribó $\dagger$}

Universitat de Barcelona, ICCUB, IEEC-UB, Barcelona, Spain

E-mail: mribo@fqa.ub.edu

\begin{abstract}
Gamma-ray binaries, which contain a massive star and a compact object, are very interesting astrophysical laboratories because particle acceleration and radiation/absorption mechanisms are modulated by the orbital phase. However, only 8 of such sources are currently known: 4 composed of an O-type star and 4 of a Be-type star, being the systems with an O-type star runaways with respect to their environment. Gaia DR2 provides us with useful information of positions, proper motions and distances for 1332 million sources. To search for new gamma-ray binaries we have studied a sample of 370 O-type stars from the GOSC catalog. In Gaia DR2 all sources are treated as single stars. Therefore, we could expect bad astrometric solutions for some gamma-ray binaries because Gaia DR2 does not fit the 7 orbital parameters for binaries. We have used the $R U W E$ quality indicator provided by Gaia DR2 to distinguish between bad astrometric solutions $(R U W E>1.15)$ and good astrometric solutions ( $R U W E \leq 1.15)$. Among the 370 O-type stars we have found 36 with bad astrometric solutions, some of which could be gamma-ray binaries. We have also computed the peculiar velocities of the remaining 334 O-type stars and found 74 runaways, some of which could also be part of new gamma-ray binaries. We conclude with a short discussion and an outlook of future work.
\end{abstract}

High Energy Phenomena in Relativistic Outflows VII - HEPRO VII

9-12 July 2019

Facultat de Física, Universitat de Barcelona, Spain

\footnotetext{
${ }^{*}$ Speaker.

${ }^{\dagger}$ Serra Húnter Fellow.
} 


\section{Introduction}

Gamma-ray binary systems are a particular type of X-ray emitting binaries composed of a compact object orbiting around a massive star and distinguished by their emission peak beyond $1 \mathrm{MeV}$ in the non-thermal spectral energy distribution. We only know 8 of such systems: 4 composed of an O-type star and 4 of a Be-type star. Since they perform as astrophysical laboratories because their particle acceleration and absorption/radiation mechanism are modulated by their orbital phase, it is important to discover new similar systems. This work has the aim to search for new gamma-ray binary systems. To do that we have used a sample of 370 O-type stars from the GOSC catalog [1][2] and their astrometric and photometric parameters from Gaia DR2 [3][4]. We have developed two different studies. In the first one we have analyzed the Gaia DR2 goodness of fit. This catalog uses a 5-parameter solution (position, parallax and proper motion) and does not take into account the eventual 7 orbital parameters of a binary system when it fits the astrometric model. For this reason we expect a bad-behaved solution for stars belonging to binary systems. The other study has focused on the runaway nature of gamma-ray binaries. The evolution of these systems begins with two Main Sequence massive stars in a circular orbit. The most massive star evolves faster until it collapses and forms a neutron star (NS) compact object after its explosion as a supernova (SN). This explosion gives a kick to the binary system that can be symmetric or asymmetric, and can disrupt it depending on the explosion [5]. Due to this kick, the massive optical star can reach a high peculiar velocity with respect to its environment and became a runaway star. We have analyzed the peculiar velocity of the stars of our sample in order to determine if they can be considered as runaway stars.

\section{The Catalog}

To do our study we have started by using the 618 early type stars from the GOSC catalog [1][2]. We have cross-matched them with the data from Gaia DR2 in order to obtain their astrometric parameters. In this process we have lost some stars which do not appear in Gaia or have duplicated source_id. We also have removed stars with multiple component, according to the GOSC catalog information, and also the stars with spectral type later than B2. Our first study consists on searching for bad-behaved solutions that could be due to stars belonging to binary systems. However, we do not want to find those bad-behaved solutions because of large errors in the parallaxes, saturation or other bad parameters. Therefore, we cleaned our sample to obtain stars with relatively reliable astrometric data, which is also good for our second study. To do that we have applied the following quality cuts: 5 parameter solution, $G \geq 6$, visibility_periods_used $\geq 10$ [6], mean_varpi_factor_al $\in[-0.23,0.32]$, $\mid$ parallax_over_error $\mid \geq 5$ [7] and positive parallaxes. Finally, we have obtained a sample of 370 O-type stars, located at distances between 0.1 and $6.2 \mathrm{kpc}$ and in a Galactic latitude range between $-17^{\circ}$ and $+13^{\circ}$. Their $G$ magnitude distribution is between 6 and 14 and it is centered around the value 9 (see Figure 1). Most of the stars are located in clusters or in associations, which are crowded star forming regions.

\section{Analysis of the Goodness of fit}

As we have explained in the Introduction, Gaia DR2 [3][4] does not take into account the 7 


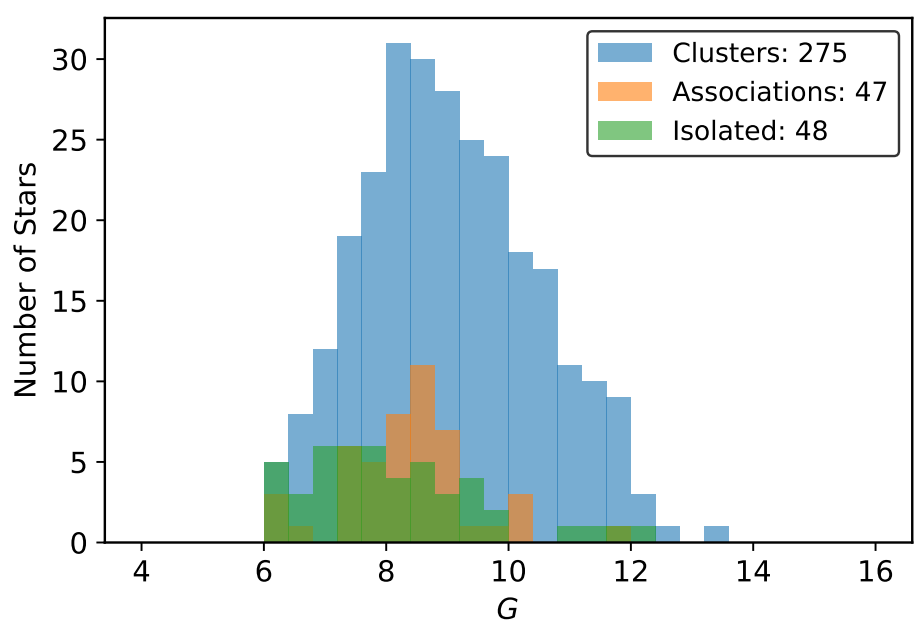

Figure 1: $G$ distribution for the sample of 370 O-type stars. We show the number of stars that are located in clusters, associations or that are isolated stars according to the information provided by the GOSC catalog.

orbital parameters to fit binary systems. For this reason, we expect bad-behaved solutions for some binaries. Gaia DR2 provides us with several goodness of fit indicators that allow us to determine the quality and reliability of the astrometric data and to distinguish between well- and bad-behaved solutions. The most useful indicators are the Unit Weight Error $(U W E)$ and the Renormalized Unit Weight Error (RUWE), both Gaussian distributions centered at a value of 1 [8]. They are defined as:

$$
U W E=\sqrt{\frac{\chi^{2}}{v}}, \quad R U W E=\frac{U W E}{U_{0}(G, C)},
$$

where $\chi^{2}$ is the along scan direction chi-square statistic, $v$ is the number of degrees of freedom and $U_{0}$ is a tabulated value that depends on $G$ magnitude and the color index of the star. As the $U W E$ indicator depends on the $G$ magnitude and the color index, we have focused on the results obtained with the $R U W E$ indicator. In Figure 2 we show both distributions for the 370 O-type stars. The values around 1 correspond to well-behaved solutions, while the values located at the tail of the distribution correspond to bad-behaved solutions. In order to separate both results, we have followed the method described in [8] but using a different sample more similar to the one of our O-type stars. We have obtained a threshold equal to 1.15 for the $R U W E$ indicator, which means that values higher than this limit correspond to bad-behaved solutions. In our sample of 370 O-type stars, 36 of them have $R U W E>1.15$, i.e, they have bad-behaved solutions. Some of them could be gamma-ray binary systems.

\section{Searching for Runaway stars}

O-type massive stars that belong to gamma-ray binaries present high peculiar velocities with respect to their environment [9][10][11]. We have analyzed the velocities of the stars from our sample in order to search for runaway stars. Taking into account the results of the previous section, 


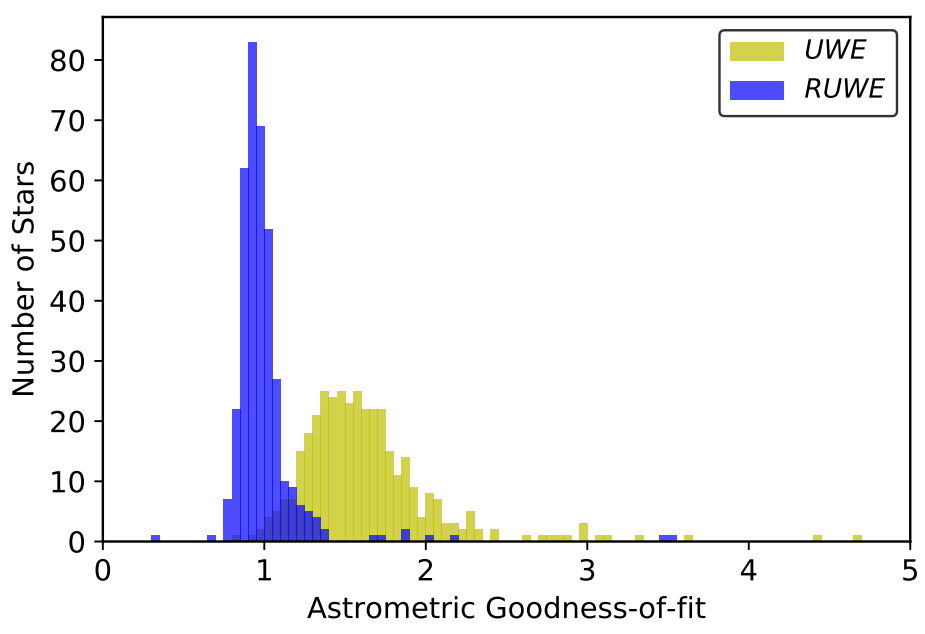

Figure 2: $U W E$ and $R U W E$ distributions for the 370 O-type stars.

we have only considered the stars with a well-behaved solution $(R U W E \leq 1.15)$. Therefore, we have only analyzed a sample of 334 O-type stars. The A5 model from [12] provides us with the values of the peculiar velocity of the Sun relative to the Local Standard of Rest (LSR). The same model gives us the information of the Galactic rotation curve. Using the transformations in [13], we have obtained the peculiar velocity of the stars with respect to their Regional Standard of Rest (RSR):

$$
V_{\mathrm{pec}}=\sqrt{U_{\mathrm{RSR}}^{2}+V_{\mathrm{RSR}}^{2}+W_{\mathrm{RSR}}^{2}}
$$

where $U_{\mathrm{RSR}}$ is positive toward the Galactic center, $V_{\mathrm{RSR}}$ is defined toward the direction of the Galactic rotation and $W_{\mathrm{RSR}}$ is positive toward the North Galactic Pole (NGP). To calculate the peculiar velocity we need to know the radial velocity of the stars, but Gaia DR2 only provides us with this value for $6 \%$ of the stars in our 334 O-type stars sample. To deal with this problem we have imposed a heliocentric radial velocity that minimizes the peculiar velocity with respect to the $\mathrm{RSR}^{1}$. This assumption affects mainly the velocity components in the Galactic Plane because our 334 O-type stars are basically at small Galactic latitudes. In order to separate the radial velocity contribution in the plane components, we have defined a new reference system formed by one component perpendicular to the line of sight direction $\left(V_{\mathrm{TAN}}\right)$ and another component in the line of sight direction $\left(V_{\mathrm{RAD}}\right)$. These two components are referred to the RSR and they are calculated as a rotation of the $\left(U_{\mathrm{RSR}}, V_{\mathrm{RSR}}\right)$ coordinates. The third component $\left(W_{\mathrm{RSR}}\right)$, perpendicular to the

\footnotetext{
${ }^{1}$ Note that this value is not zero. In fact, for stars in the Galactic Plane (where we assume that the Sun is located), it corresponds to the heliocentric radial velocity of the RSR measured from the LSR, considering the chosen Galactic rotation curve, plus the radial velocity of the LSR as seen from the Sun. For stars above and below the Galactic Plane, the chosen heliocentric radial velocity to minimize the peculiar velocity has a component outside of the Galactic Plane, and thus it is slightly different (but never more than $5 \mathrm{~km} \mathrm{~s}^{-1}$ ) than the heliocentric radial velocity of the RSR measured from the LSR, considering the chosen Galactic rotation curve, plus the radial velocity of the LSR as seen from the Sun. This is a conservative assumption.
} 


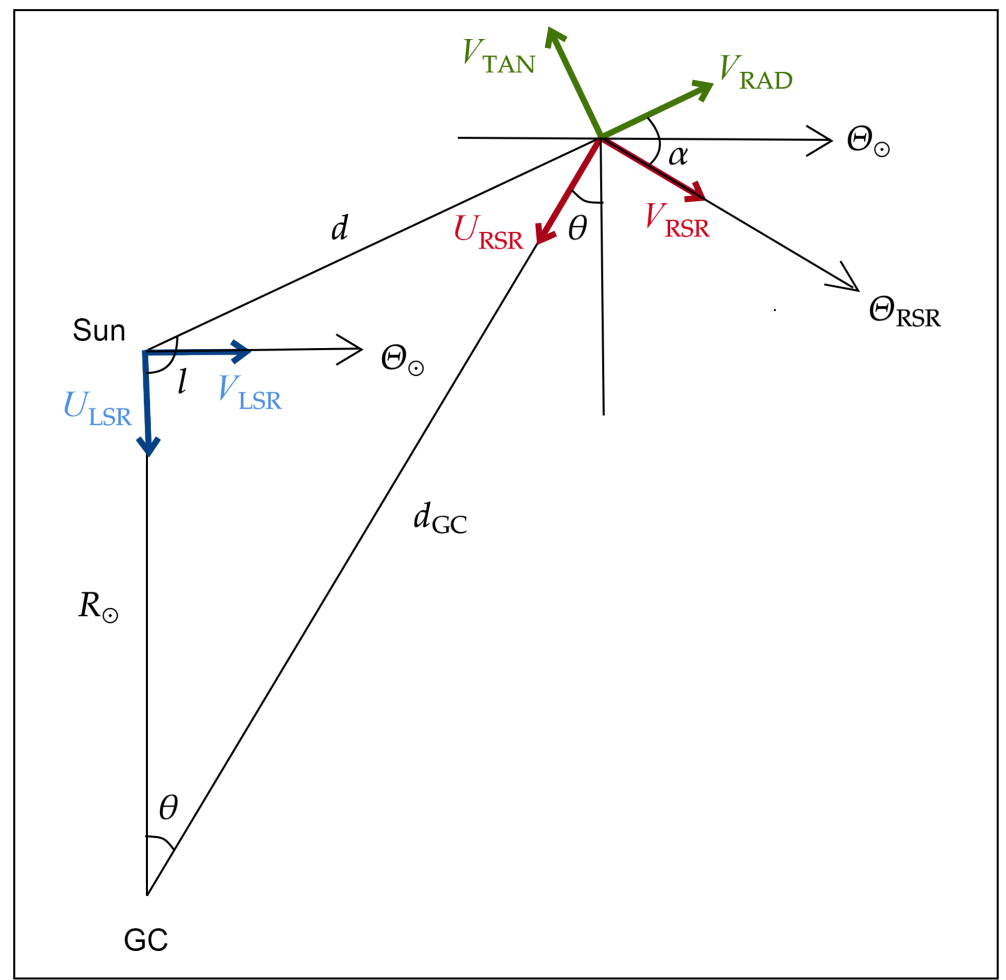

Figure 3: Reference systems in the Galactic Plane ( $W_{\mathrm{LSR}}$ and $W_{\mathrm{RSR}}$ velocities are perpendicular to it).

Galactic Plane, is not affected by this change of reference system. In Figure 3 we show a diagram of the reference systems used in this study.

The advantage of using this new reference system is that the $V_{\mathrm{RAD}}$ component is zero for stars located in the Galactic Plane and typically smaller than $5 \mathrm{~km} \mathrm{~s}^{-1}$ for stars at small Galactic latitudes (see footnote 1). Therefore, to do our study we have used a 2-D approach ( $V_{\mathrm{TAN}}, W_{\mathrm{RSR}}$ ). Our 334 O-type star distribution is composed of field stars, which define the environment velocity, and runaway stars, which have significant peculiar velocities with respect to their environment. In order to determine a criterion to separate the field stars from the runaway stars, we have defined the following ellipse:

$$
E=\frac{\left(V_{\mathrm{TAN}}-\mu_{\mathrm{V}_{\mathrm{TAN}}}\right)^{2}}{\left(3 \cdot \sqrt{\sigma_{\mathrm{V}_{\mathrm{TAN}}}^{2}+\sigma_{\mathrm{V}_{\mathrm{TAN} *}}^{2}}\right)^{2}}+\frac{\left(W_{\mathrm{RSR}}-\mu_{\mathrm{W}_{\mathrm{RSR}}}\right)^{2}}{\left(3 \cdot \sqrt{\sigma_{\mathrm{W}_{\mathrm{RSR}_{\mathrm{d}}}^{2}}^{2}+\sigma_{\mathrm{W}_{\mathrm{RSR} *}}^{2}}\right)^{2}}
$$

where $\left(\mu_{\mathrm{V}_{\mathrm{TAN}}}, \mu_{\mathrm{W}_{\mathrm{RSR}}}\right)$ and $\left(\sigma_{\mathrm{V}_{\mathrm{TAN}_{\mathrm{d}}}}, \sigma_{\mathrm{W}_{\mathrm{RSR}_{\mathrm{d}}}}\right)$ are the mean and the standard deviation of Gaussian fits to the velocity distributions, respectively, and $\left(\sigma_{\mathrm{V}_{\mathrm{TAN}_{*}}}, \sigma_{\mathrm{W}_{\mathrm{RSR}}}\right)$ are the uncertainties computed for the velocities of each star. All stars that are outside the surface defined by this ellipse $(E>1)$ have been classified as runaway stars while the stars within the surface of this ellipse $(E \leq 1)$ have been classified as field stars. In Figure 4 we show the velocities in the 2-D approach of our 334 O-type stars sample. We observe that the field stars are located around the origin while the runaway stars are roughly outside a centered ellipse.

We show in Figure 5 the Aitoff projection in Galactic coordinates of our 334 O-type stars 


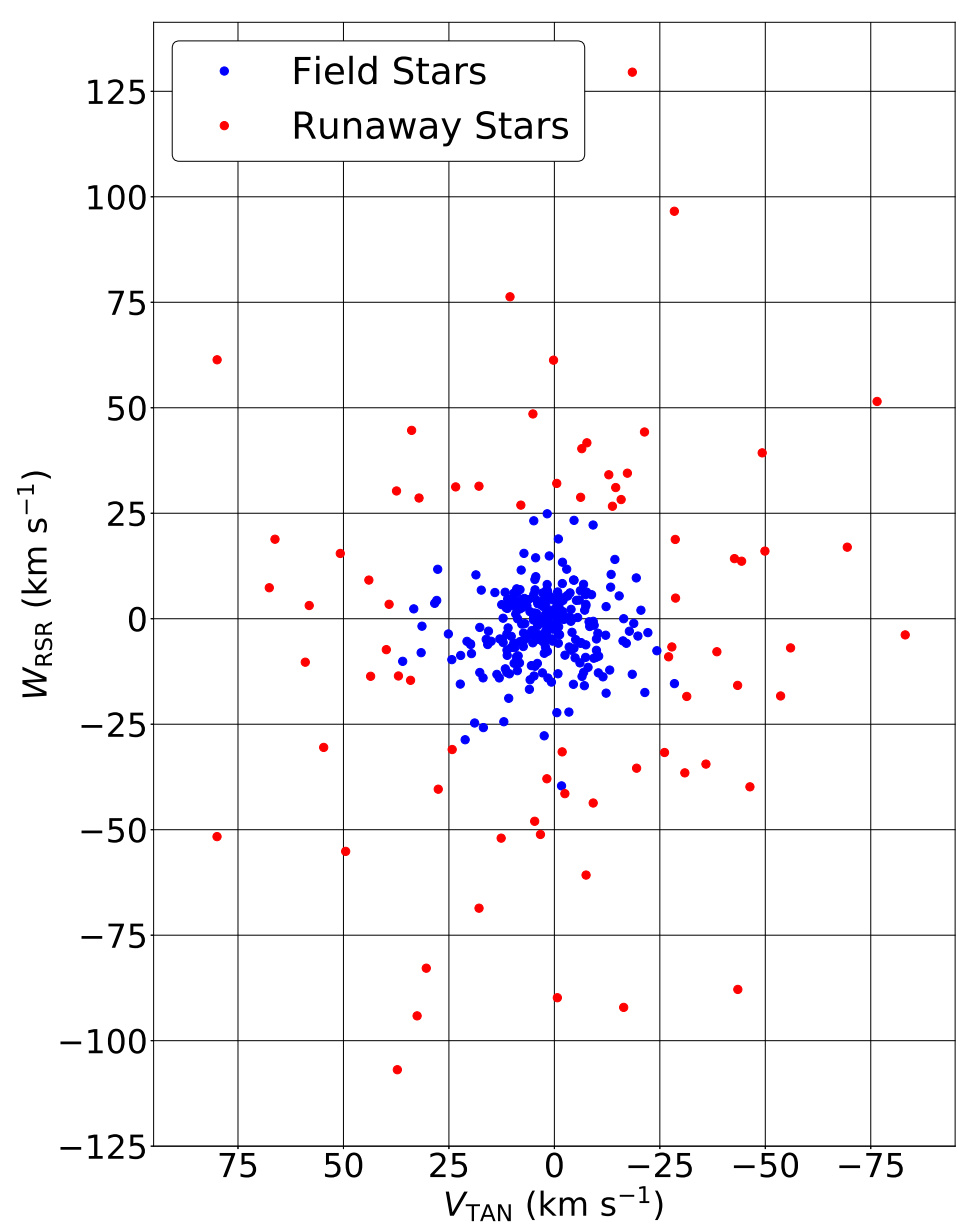

Figure 4: Distribution of $V_{\mathrm{TAN}}$ vs $W_{\mathrm{RSR}}$ for the sample of 334 O-type stars with $R U W E \leq 1.15$.

sample. Note that the dispersion of the runaway stars with respect to the Galactic Plane is higher than the dispersion of the field stars.

From a total of 334 stars, we have obtained 74 runaway stars, with peculiar velocities between 28 and $133 \mathrm{~km} \mathrm{~s}^{-1}$. Some of them could be gamma-ray binaries.

\section{Discussion and Outlook}

The formation of gamma-ray binary systems takes place in the forming region close to the Galactic Plane. This is determined by the O-type field star region (OFSR), where field stars remain during the span of their lives. The characteristic lifetime of the gamma-ray binary phase is at most of the order of $10^{5}$ years [14]. For this reason, the candidates to be gamma-ray binaries had to be 


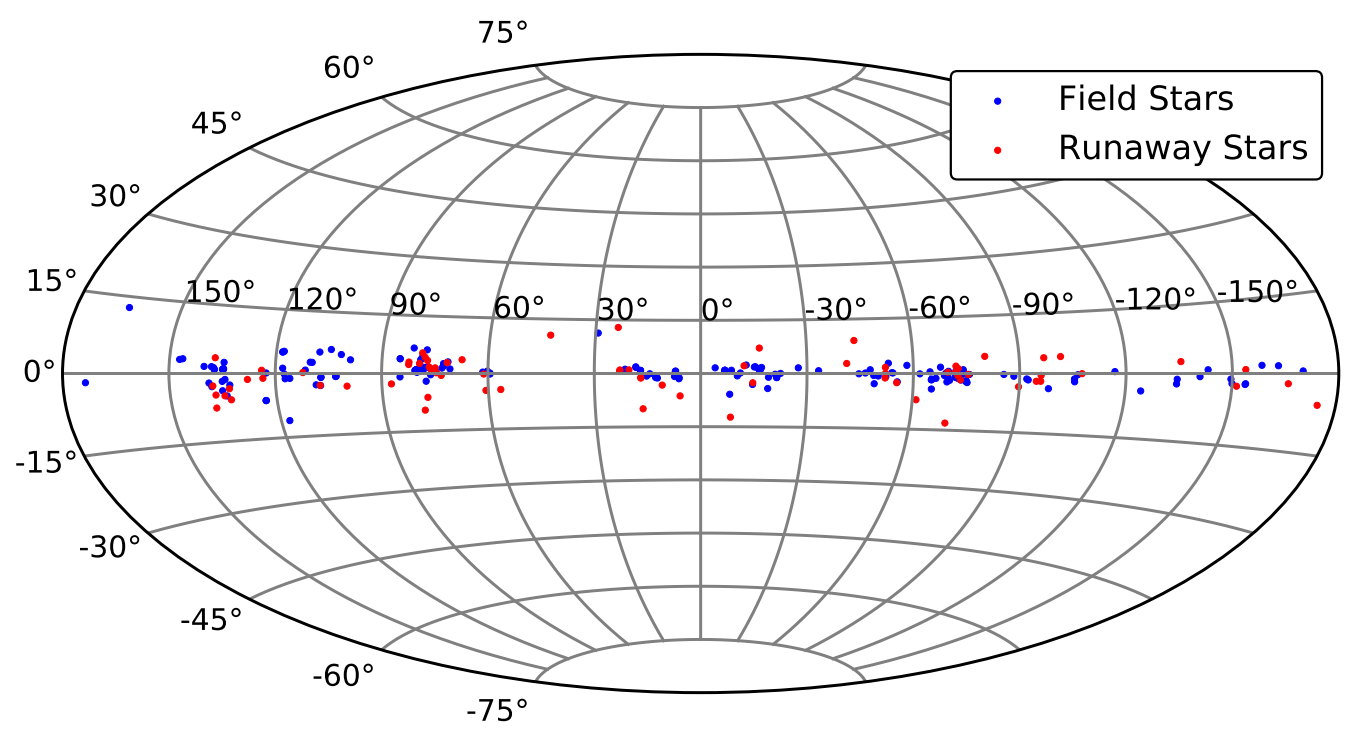

Figure 5: Aitoff projection in Galactic coordinates of the 334 O-type stars with $R U W E \leq 1.15$.

located in the OFSR in the last $10^{5}$ years. Among the 74 runaway stars, 61 were within the OFSR in the last $10^{5}$ years, therefore, they could be gamma-ray binary systems. From the 74 stars, 24 are located in the H.E.S.S. Galactic Plane Survey area [15] and 2 are within the 95\% c.l. contours of a source listed in the $4^{\text {th }}$ Fermi-LAT source catalog [16]. One of these 2 stars belongs to the known gamma-ray binary system LS 5039.

Among the 36 stars with bad astrometric solutions, all of them were in the OFSR in the last $10^{5}$ years, 11 are located in the H.E.S.S. Galactic Plane Survey area and 2 are within the $95 \%$ of c.l. contours listed in the $4^{\text {th }}$ Fermi-LAT source catalog.

The outlook for future research is the study of these gamma-ray binary candidates. It will be necessary to search for non-thermal emission in other catalogs, measure the radial velocities and search for radial velocity variability to unveil possible orbital motion. These are future targets for CTA. In addition, similar studies could be conducted with databases of Be stars, for which Gaia DR3 could also provide relevant information regarding emission line features.

\section{Acknowledgments}

We thank an anonymous referee for useful comments that helped to improve the content of this manuscript. 
The authors acknowledge partial support by the Spanish Ministerio de Ciencia, Innovación y Universidades (MICINN) under grant FPA2017-82729-C6-2-R (AEI/FEDER, UE).

This work has made use of data from the European Space Agency (ESA) mission Gaia (https://www.cosmos.esa.int/gaia), processed by the Gaia Data Processing and Analysis Consortium (DPAC, https://www.cosmos.esa.int/web/gaia/dpac/consortium). Funding for the DPAC has been provided by national institutions, in particular the institutions participating in the Gaia Multilateral Agreement.

\section{References}

[1] J. Maíz Apellániz, A. Sota, N. I. Morrell, R. H. Barbá, N. R. Walborn, E. J. Alfaro et al., First whole-sky results from the Galactic $O$-Star Spectroscopic Survey, in Massive Stars: From $\alpha$ to $\Omega$, p. 198, June, 2013, 1306.6417.

[2] J. Maiz-Apellaniz, N. R. Walborn, H. A. Galue and L. H. Wei, A galactic O star catalog, The Astrophysical Journal Supplement Series 151 (2004) 103.

[3] Gaia Collaboration, Prusti, T., de Bruijne, J. H. J., Brown, A. G. A., Vallenari, A., Babusiaux, C. et al., The Gaia mission, A\&A 595 (2016) A1.

[4] Gaia Collaboration, A. G. A. Brown, A. Vallenari, T. Prusti, J. H. J. de Bruijne, C. Babusiaux et al., Gaia Data Release 2. Summary of the contents and survey properties, A\&A 616 (2018) A1 [1804.09365].

[5] A. Blaauw, On the origin of the $O$ - and B-type stars with high velocities (the "run-away" stars), and some related problems, BAN 15 (1961) 265.

[6] L. Lindegren, J. Hernández, A. Bombrun, S. Klioner, U. Bastian, M. Ramos-Lerate et al., Gaia Data Release 2. The astrometric solution, A\&A 616 (2018) A2 [1804.09366].

[7] X. Luri, A. G. A. Brown, L. M. Sarro, F. Arenou, C. A. L. Bailer-Jones, A. Castro-Ginard et al., Gaia Data Release 2. Using Gaia parallaxes, A\&A 616 (2018) A9 [1804.09376].

[8] L. Lindegren, "Re-normalising the astrometric chi-square in Gaia DR2." August, 2018, GAIA-C3-TN-LU-LL-124.

[9] Ribó, M., Paredes, J. M., Romero, G. E., Benaglia, P., Martí, J., Fors, O. et al., LS 5039: A runaway microquasar ejected from the galactic plane, A\&A 384 (2002) 954.

[10] J. Moldón, M. Ribó, J. M. Paredes, W. Brisken, V. Dhawan, M. Kramer et al., On the origin of LS 5039 and PSR J1825-1446, A\&A 543 (2012) A26.

[11] Marcote, B., Ribó, M., Paredes, J. M., Mao, M. Y. and Edwards, P. G., Refining the origins of the gamma-ray binary 1FGL J1018.6-5856, A\&A 619 (2018) A26.

[12] M. J. Reid, K. M. Menten, A. Brunthaler, X. W. Zheng, T. M. Dame, Y. Xu et al., Trigonometric Parallaxes of High Mass Star Forming Regions: The Structure and Kinematics of the Milky Way, APJ 783 (2014) 130 [1401.5377].

[13] D. R. H. Johnson and D. R. Soderblom, Calculating galactic space velocities and their uncertainties, with an application to the Ursa Major group, AJ 93 (1987) 864.

[14] G. Dubus, Gamma-ray binaries and related systems, The Astronomy and Astrophysics Review 21 (2013). 
[15] H. E. S. S. Collaboration, H. Abdalla, A. Abramowski, F. Aharonian, F. Ait Benkhali, E. O. Angüner et al., The H.E.S.S. Galactic plane survey, A\&A 612 (2018) A1 [1804. 02432 ].

[16] The Fermi-LAT Collaboration, Fermi Large Area Telescope Fourth Source Catalog, arXiv e-prints (2019) arXiv:1902.10045 [1902.10045]. 